\title{
The Galactic System of Open Star Clusters: A Personal Perspective
}

\author{
Randy L. Phelps \\ Department of Physics and Astronomy, California State University, \\ Sacramento, 6000 J Street, Sacramento, CA 95819, USA
}

\begin{abstract}
Technology is now allowing for the investigation of star clusters outside of the Milky Way. As attention turns to the extragalactic star clusters, a perception that the system of star clusters in the Milky Way is well understood may grow, resulting in the neglect of these important objects. In this review, the status of our understanding of the Milky Way's open star cluster population will be discussed. Specifically, I will attempt to illustrate not only the important information that can and must be learned from these nearby star clusters, but also the degree to which our understanding of the Galactic open clusters remains incomplete.
\end{abstract}

\section{Introduction}

The advent of adaptive optics on large-aperture (6-10 meter diameter) groundbased telescopes, at sites with good seeing, along with space telescopes that routinely obtain high angular resolution (e.g., the Hubble Space Telescope), is pushing the study of star clusters beyond the Milky Way, into the Local Group and beyond. This trend will continue as high-resolution, ground-based imaging becomes routine, and new space-based telescopes like the Next Generation Space Telescope (NGST) become available.

As attention turns to the extragalactic star clusters, a perception that the system of star clusters in the Milky Way is well understood may grow, resulting in the neglect of these important objects. In this review, the status of our understanding of the Milky Way's open star cluster population will be discussed. Specifically, I will attempt to illustrate not only the important information that can and must be learned from these nearby star clusters, but also the degree to which our understanding of the Galactic open clusters remains incomplete.

\section{What We Have to Work With}

Even after 14 years, the basic catalog of open cluster remains the "Lund Catalog of Open Cluster Data" (Lyngå 1987), although the online "WebDA" database (Mermilliod 2001) in many ways serves as a more modern replacement. The Lund catalog contains some 1200 or so catalogued clusters, with many having little or no information available for them. Figure 1 shows a portion of the Lund catalog, illustrating the incompleteness inherent in what remains one of the most comprehensive catalogs of open cluster data. Additionally, the vast 


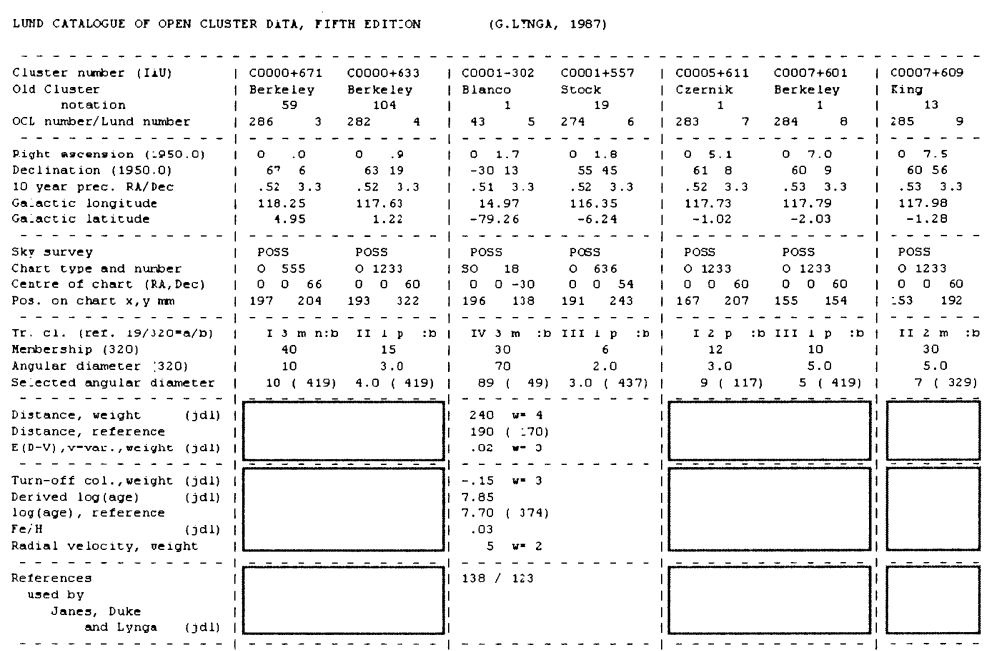

Figure 1. A portion of the Lund "Catalog of Open Cluster Data"

majority of open clusters have no modern CCD photometry available. As we begin to investigate star clusters in other galaxies, it is important to realize that our understanding of the Milky Way's open cluster population, often taken for granted, is seriously incomplete.

As a wide range of astrophysical problems are studied with open clusters (e.g., the formation and evolution of the Galactic disk, the formation of perhaps the majority of stars, and the properties of peculiar or otherwise interesting stars), it is distressing to realize that basic properties like distance and age are unknown for a large fraction of catalogued clusters. As future photometric studies may well provide the bulk of the information available for extragalactic clusters, it is instructive to review what is known of the Galaxy's open cluster population from photometry alone.

\subsection{The Ideal World}

It is not surprising that the open clusters that have been observed with CCDs tend to be those that are already among the best-studied clusters. Studies of key open clusters, for example the very old, metal rich cluster NGC 6791 (Montgomery, Janes \& Phelps 1994) and the well-known solar-age cluster M67 (Montgomery, Marschall \& Janes 1993) have benefited enormously from the precise nature of CCD photometry for hundreds/thousands of stars. Studies of younger clusters (e.g., Phelps \& Janes 1994, Keller et al. 2001) have also benefited from CCD studies, by revealing the shape of the cluster sequences to low masses, thus allowing for better constraints on cluster distances to be obtained. In many cases to-date, cluster sequences in the resulting color-magnitude diagrams (CMDs) are fairly well-defined (Figures 2 and 3), a result of the richness and/or proximity of the clusters to the Sun.

CMDs like those shown in Figures 2 and 3 represent something of a "best case scenario" for open cluster studies, for barring detailed information on radial velocities and proper motions, there is no easy way to remove field stars from 

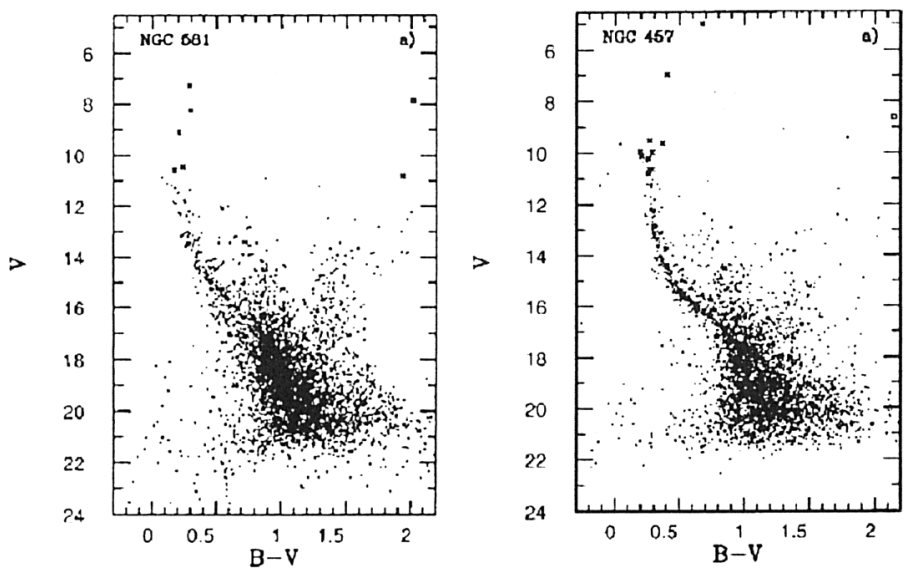

Figure 2. CMDs for two young open clusters (from Phelps \& Janes, 1994)

the CMD. As field stars often overwhelm cluster stars, at faint magnitudes in particular, uncertainties will remain when determining vital cluster parameters like distance and age. The WIYN Open Cluster Study, or WOCS, (Mathieu 2000 ) is the sole, large-scale undertaking to "do things right", by means of a comprehensive photometric, radial velocity and proper motion study of key open clusters. The work involved is enormous, however, and the WOCS will study only a handful of clusters. Additionally, several photometric surveys are currently being undertaken to obtain multicolor CCD photometry of open clusters. The CFHT Open Cluster Survey (Kalirai et al. 2001), for example, will obtain deep (V 25 mag) photometry of 19 open clusters. Groups in India (e.g., Subramaniam \& Sagar 1999), Korea (e.g., Lee, Kang \& Ann 1999), Italy (e.g., Sandrelli et al. 1999), Argentina (e.g., Piatti, Clariá \& Bica 2000) to name but a few, are also undertaking photometric surveys, to varying levels.

For "old" open clusters, here defined to be those as old or older than the Hyades with an age of $625 \mathrm{Myr}$ (Perryman et al. 1998), numerous photometric CCD studies have been undertaken to improve upon our knowledge of clusters in this important age range. Among the groups that have worked extensively on these clusters are those of Carraro et al. (e.g., Carraro et al. 1994), Kaluzny et al. (e.g., Kaluzny 1994a) and Twarog et al. (e.g., Twarog, Anthony-Twarog $\&$ Hawarden 1995). These groups have been at the forefront of efforts to derive reliable ages and distances of the old open clusters by means of isochrone matches to the derived CMDs (Figure 4). The old open clusters are especially important as they span the age of the Galactic disk, and hence they are crucial tools for studying the temporal, spatial and chemical evolution of the Galactic disk. For this reason, the "old" open clusters are the subjects of the remainder of this review. 

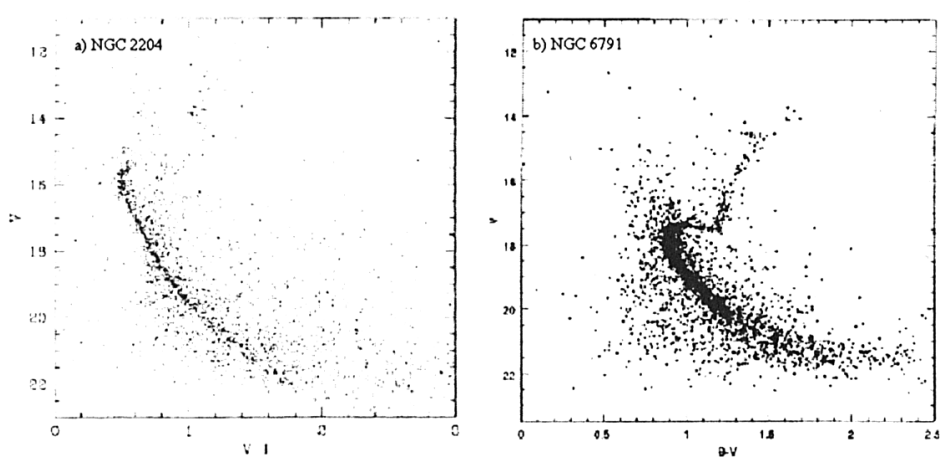

Figure 3. CMDs for two old open clusters (from Kassis et al. 1997 and Montgomery et al. 1994)
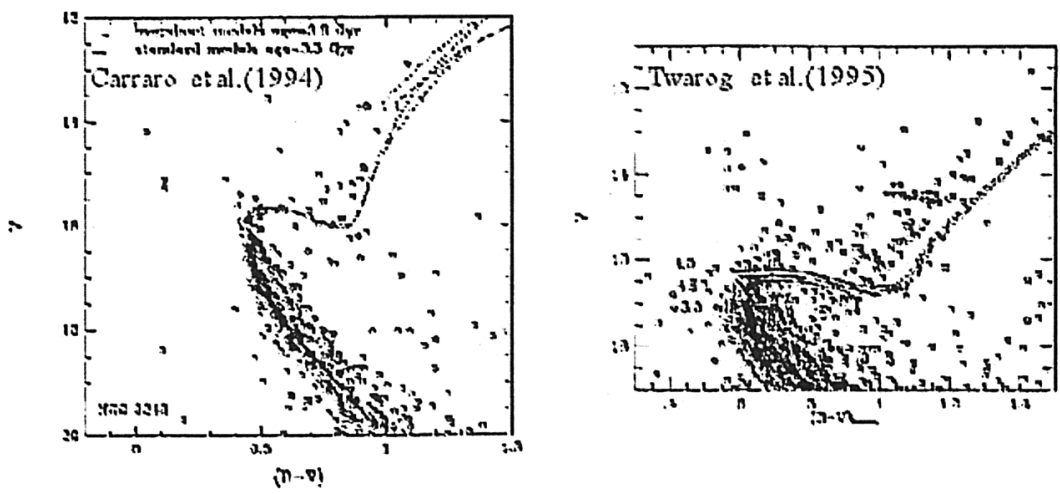

Figure 4. Isochrone matches to the CMDs of NGC 2243 and Melotte 66 (from Carraro et al. 1994 and Twarog et al. 1995) 


\subsection{The Reality}

A significant fraction of the now-known old open clusters, and most younger ones for that matter, are distant, highly reddened objects. Their observed CMDs, therefore, are poorly defined, or "scruffy", compared to those derived for the best-case-scenario clusters. Figure 5, for example, shows a collection of CMDs, derived during a photometric search for old open clusters by Phelps, Janes \& Montgomery (1994). Despite the "scruffy" nature of these diagrams, a great deal of useful information can be derived, although one must be careful to not over interpret the CMDs. Care must be exercised when quoting values for distance and age, for example, and the large uncertainties that are involved must be taken into account when using these values for subsequent investigations.

Isochrone matches, for example, are not always possible when estimating ages and distances of most old open clusters. A morphological index, however, can provide a reasonably robust means to estimate relative ages of old clusters (e.g., Anthony-Twarog \& Twarog 1985; Buonanno, Corsi \& Fusi Pecci 1989; Sarajedini \& Demarque 1990; VandenBerg, Bolte \& Stetson 1990; Janes \& Phelps 1994). Janes \& Phelps (1994), for example, derived two parameters, $\delta \mathrm{V}$ and $\delta 1$, for such a purpose. The parameter $\delta \mathrm{V}$ measures the difference in luminosity between the main sequence turnoff (defined by the apparent magnitude of the inflection point in the subgiant branch) and the luminosity of the red giant clump. The parameter $\delta 1$ measures the difference in color between the main sequence turnoff and a point on the red giant branch located $1 \mathrm{mag}$ brighter than the main sequence turnoff. As $\delta \mathrm{V}$ increases with cluster age, while $\delta 1$ decreases with cluster age, these parameters provide a means to estimate relative cluster ages.

Janes \& Phelps (1994) provided a calibration of $\delta \mathrm{V}$ with published isochrone ages of clusters, to derive the so-called Morphological Age Index, or MAI. As pointed out in Janes \& Phelps (1994), however, the calibration of the MAI is based on published isochrone ages that used various isochrones (and hence input physics). As noted in their paper, the MAI should not be considered a true cluster age. A recent study (Tosi et al. 1998), for example, indicates that the MAI tends to overestimate actual cluster ages, although it must be stressed that the MAI was derived for the sole purpose of providing not absolute, but relative, cluster ages. A recalibration of the MAI, using self-consistent isochrone ages, is currently underway (Phelps in preparation).

\section{What We Can Learn from Photometric Studies of Old Open Clusters}

Despite the limitations imposed by nature regarding the often poorly defined cluster CMDs, there is a great deal of knowledge that can be gained from open cluster studies, provided one does not overinterpret the available information. A prime area for investigation, and one that has heavily used open clusters for example, is the formation and evolution of the Galactic disk. 

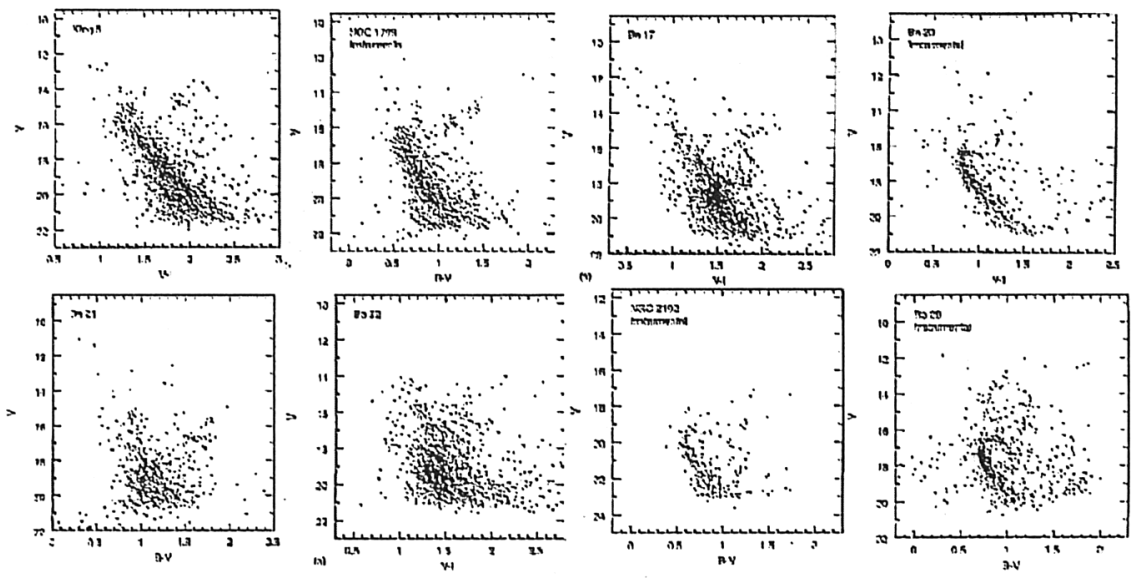

Figure 5. CMDs for old open clusters (from Phelps, Janes \& Montgomery 1994)

\subsection{The Oldest Open Cluster}

The large-scale literature and photometric search for open clusters undertaken by Phelps et al. (1994), while a number of years old now, remains the most comprehensive single search for old open clusters to-date. From that study, some 75 clusters were found to be as old, or older than the Hyades, while some 15-19 clusters were found to be as old, or older than the solar-age cluster M67. Since that study, a number of individual studies have revealed more old open clusters. Table 1 lists clusters discovered or otherwise confirmed to be old since the Phelps et al. (1994) study, listing the cluster name, the adopted age and the reference.

Table 1. Newly Identified Old Open Clusters

\begin{tabular}{lcl}
\hline Cluster Name & Age (Gyr) & \multicolumn{1}{c}{ Reference } \\
\hline Berkeley 18 & 4 & Carraro et al. (1999b) \\
Berkeley 66 & $3-4$ & Phelps (1996) \\
ESO 093-SC08 & $4-5$ & Bica, Ortolani \& Barbuy(2000) \\
Hafner 6 & 1 & Patat \& Carraro(1995a) \\
NGC 2539 & 0.6 & Lapasset, Claria \& Mermilliod(2000) \\
NGC 7762 & 2 & Patat \& Carraro(1995b) \\
Pismis 3 & 2 & Carraro \& Ortolani(1994) \\
Tombaugh 1 & 1 & Carraro \& Patat(1995) \\
\hline \hline
\end{tabular}

Janes \& Phelps (1994) used the MAI to investigate not only the relative ages of Galactic open clusters, but also the relative ages of Galactic open and globular clusters. Based on this information, it appeared as though a single open cluster, Berkeley 17, or Be 17, has an age greater than the youngest globular clusters. Subsequent, more detailed studies of Be 17 (Kaluzny 1994b, Phelps 1997, Carraro et al.1999a) have confirmed an old age for the cluster, but its 

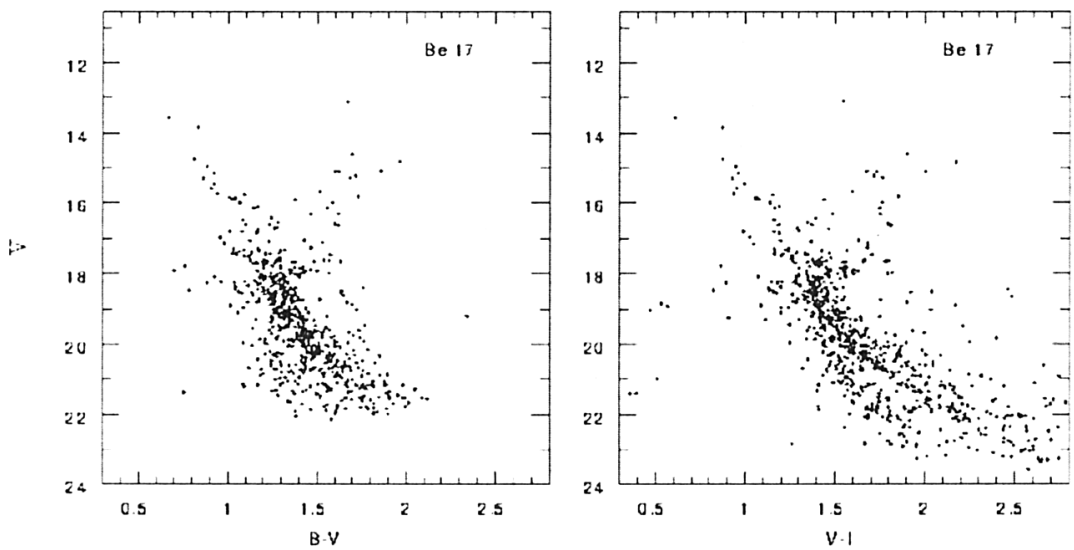

Figure 6. $\quad \mathrm{BV}$ and VI CMDs for the old open cluster, Berkeley 17 (from Phelps 1997)

distinction as the oldest known open cluster remains controversial. The age of Be 17 is especially important to determine, as it potentially places a constraint on the age of the Galactic disk.

Figure 6, taken from Phelps (1997), shows the BV and VI CMDs of Be 17. Making no assumptions on the reddening and metallically, Phelps (1997) derived an age for Be 17 of 10-13 Gyr, based on isochrone matches to the CMDs. Although a metallicity estimate was available from the literature (Friel 1995), the photometry provided for that study is now known to have a zero point error, and the metallicity was deemed to be uncertain. Kaluzny (1994b), based on a comparison of the Be $17 \mathrm{CMD}$ to that of the metal rich cluster NGC 6791, derived an age of 9 Gyr for Be 17. By adopting the Friel (1995) metallicity of $-0.29 \pm 0.13$ dex, Carraro et al. (1999a) derived an age of $9 \pm 1$ Gyr for Be 17, lower than, but still within the errors quoted by Phelps (1997). Carraro et al. (1999a) also reanalyzed Be 17 using the technique of Phelps (1997) and found that an age of $11 \pm 1 \mathrm{Gyr}$ "cannot be firmly excluded because of the many uncertainties affecting stellar models in the mass range 1.0 to 1.5 solar masses".

The case of Be 17 illustrates an important point that is often overlooked in open cluster studies, which is that matches of isochrones to CMDs are really not numerical fits, but rather judgements by various investigators as to what constitutes a best "fit". Discrepancies result from differences in judgement, as well as from the fact that field star contamination, and high and often differential reddening make isochrone matches difficult. This need not mean that the information open clusters provide is not useful, but rather it indicates a need to appreciate the inherent uncertainties one faces when obtaining information from open clusters!

Being careful to not overinterpret the information open clusters can provide, what can one learn from the photometric ages and distances derived for open clusters? For more information regarding the information available from chem- 
ical composition studies for old open clusters, the reader is referred to reviews by Friel (1995) and Phelps (2000).

\subsection{Sizes of Old Open Clusters}

Of interest to investigators of extragalactic star clusters is the mean diameter or radius of Galactic open clusters. Having estimated distances, and using published angular sizes of open clusters from Lyngå (1987), Janes \& Phelps (1994) found that the old open clusters have a median diameter of $5 \mathrm{pc}$. Such a cluster subtends 1 arcmin at a distance of about $17 \mathrm{kpc}$, or 1 arcsec at a distance of about $1 \mathrm{Mpc}$.

\subsection{Scale Height of Old Open Clusters}

Phelps \& Janes (1994) also found that the scale height of old open clusters relative to the disk of the Galaxy is $375 \mathrm{pc}$. This compares to a scale height of $55 \mathrm{pc}$ for the young open clusters.

\subsection{Galactic Positions of Old Open Clusters}

It has long been recognized that old open clusters are preferentially located in the outer part of the Galactic disk (van den Bergh \& McClure 1980). There is a striking cutoff in at a Galactocentric radius of about $7.5 \mathrm{kpc}$, at which very few old clusters are found interior to that radius, and the majority are found outside of that radius. This is attributed, primarily, to the destructive nature of cluster encounters with giant molecular clouds, which themselves are located primarily in the inner Galactic disk. The spatial distribution of old open clusters contrasts markedly with that of the young open clusters, which are uniformly distributed about the solar neighborhood (see Figures 7 and 9 in Janes \& Phelps, 1994). While old clusters may exist in small numbers interior to the solar circle, the presence of young clusters there argues that the deficiency in known old clusters interior to the solar circle is not mainly a selection effect. An interesting question remains, however, as to how many old clusters may yet be found in the inner part of the Galactic disk.

There is a difference in scale height between the young $(\leq 600-800 \mathrm{Myr})$ and old ( $\geq 600-800 \mathrm{Myr}$ ) open clusters, as outlined earlier. Within the age range spanned by the old open clusters (i.e., the majority of the lifetime of the Galactic disk), and in particular for ages greater than 2-3 Gyr, there is no apparent correlation between cluster age and distance from the Galactic plane (Figure 11 of Janes \& Phelps 1994). Additionally, there is little/no apparent correlation of age with Galactocentric radius, beyond $7.5 \mathrm{kpc}$ from the Galactic center (Figure 12 of Janes \& Phelps 1994).

\subsection{Future Directions}

These observations, derived from photometric studies of "scruffy" open cluster CMDs, along with chemical abundance studies (e.g., Geisler 1987, Friel \& Janes1993, Friel 1995, Carraro, Ng \& Portinari 1998) suggest an interesting history for the Galactic disk, including potential accretion events. Some controversy remains, however, regarding the interpretation of the open cluster data, as far as the evolutionary history of the Galactic disk is concerned (e.g., Twarog 
et al. 1997). Progress will be made through a combination of detailed studies of individual clusters, and the discovery of new old clusters. For example, Tosi et al. (1998) have shown that the cluster Berkeley 21 (Be1) has a metallicity, $[\mathrm{Fe} / \mathrm{H}]$, near $-0.5 \mathrm{dex}$, rather than $-0.97 \mathrm{dex}$ as was previously reported (Friel \& Janes 1993). This type of refinement will, for example, help us to better understand the form of the age-metallicity relation and the age-Galactocentric radius relation in the Galactic disk. It is essential to undertake further detailed studies of key individual clusters to improve our understanding the evolution of the Galactic disk.

Table 2 lists the old open clusters from the Janes \& Phelps (1994) list that lie at the greatest Galactocentric radii (columns 2 and 4). Despite the increase in the number of known clusters with large Galactocentric radii, their number remains small if we are to learn about the development of the outer part of the Galactic disk. Another avenue for making progress in this area would be the identification of additional clusters in distant parts of the Galactic disk.

Table 2. Large- $\mathrm{R}_{G C}$ Old Open Clusters

\begin{tabular}{cccc}
\hline Cluster Name & $\mathrm{R}_{G C}(k p c)$ & Cluster Name & $\mathrm{R}_{G C}(k p c)$ \\
\hline King 2 & 13.0 & Berkeley 21 & 14.3 \\
\hline Tombaugh 2 & 13.1 & Berkeley 20 & 16.1 \\
\hline AM 2 & 14.1 & Berkeley 29 & 18.7 \\
\hline \hline
\end{tabular}

Table 3 lists the clusters from the Janes \& Phelps (1994) survey with the greatest ages, based on the MAI (columns 2 and 4). Nearly all of the oldest open clusters in the table were unknown (as far as their ages are concerned) as recently as 10-15 years ago. Additional insight into the formation history of the Galactic disk is likely to develop if additional extremely old open clusters, such as those listed in Table 3 , can be found.

Table 3. The Oldest Open Clusters

\begin{tabular}{cccc}
\hline Cluster Name & MAI (Gyr) & Cluster Name & MAI (Gyr) \\
\hline Berkeley 17 & 12.6 & NGC 188 & 7.2 \\
\hline NGC 6791 & 9.5 & Berkeley 32 & 7.2 \\
\hline Collinder 261 & 9.5 & Berkeley 39 & 7.2 \\
\hline AM 2 & 8.3 & & \\
\hline \hline
\end{tabular}

\section{The Palomar-Las Campanas Observatory-NOAO (PLCON) Open Cluster Survey}

To improve our understanding of the Galactic open cluster population, a largescale, systematic photometric survey of open clusters is being undertaken by the author. The Palomar-Las Campanas Observatory-NOAO (PLCON) Open Cluster Survey differs from other surveys (e.g., WOCS and the CFHT Open Cluster Survey) in that it has as its goal not the detailed study of one to two dozen clusters, but rather a "snapshot" photometric survey of one-third to onehalf of all catalogued open clusters. When completed, V and I band photometry 
will be available for up to 500 open clusters. Data for some 300 clusters are already on-hand.

The primary goal of the survey is to identify "new" old open clusters, in order to improve upon our understanding of the formation and evolution of the Galactic disk over the majority of its existence. Specifically the project seeks to identify open clusters in the outer portions of the Galactic disk, where our information is most incomplete, and to establish whether or not there are clusters as old as, or older than, Berkeley 17.

The PLCON survey will provide a systematic, homogeneous database of cluster photometry, by using a small number of telescopes (the Palomar Observatory 1.5 meter and the Las Campanas Observatory 1 meter telescopes), consistent filters at each telescope, and a uniform observing strategy. Each cluster is observed using short (typically $3 \mathrm{sec}$ ), medium (30 sec) and long (360 sec in V, $300 \mathrm{sec}$ in I) exposures in with each of the V and I filters. The short exposures are used to obtain photometry of the brightest stars, which constrain the cluster age, while the longer exposure frames are used to reach farther down the cluster sequence in the CMD, where the shape better constrains the cluster distance. The selection of the $\mathrm{V}$ and I filters was made for several reasons:

- While observations with more than two filters are highly desirable, the goal of observing several hundred clusters makes selection of only two filters necessary, particularly given the long readout time that still exists with the CCD at the Palomar telescope;

- Much of the scheduled observing time is/was during bright time, necessitating the use of "red" filters to minimize the effects of the bluer sky background;

- A primary goal of the Survey is the identification of "new" old open clusters. Their detection is enhanced by the use of redder filters since the brightest stars in old clusters are red. Additionally, interstellar reddening effects for distant clusters, located near the Galactic plane, are lessened when using redder filters.

The survey is meeting with considerable success, both in terms of obtaining quality photometry for a large number of open clusters, and in the identification of "new" old open clusters. Figure 7, for example, shows the CMDs of four newly identified old open clusters. Figure 7a shows the CMD for the cluster Berkeley 57 (Be 57). The presence of a red-giant clump at V 15.3, (V-I) 1.7 indicates Be 57 is an old cluster, with an age of $\sim 2$ Gyr, based on the MAI. Figure $7 \mathrm{~b}$ shows the CMD for the cluster Tombaugh 4 (To 4). The presence of a distinct main-sequence turnoff, and a probable red-giant clump at V 16 , (VI) 2.4 indicates that To 4 is also an old cluster, with an age of $\sim 1.5 \mathrm{Gyr}$, based on the MAI. Figure 7c shows the CMD for the Berkeley 100 (Be 100) region ( $\mathrm{x}$ 's) and an associated field region (dots). The presence of a red-giant clump at $\mathrm{V} \sim 17,(\mathrm{~V}-\mathrm{I}) \sim 2.2$ indicates Be 100 is an old cluster, with an age of $\sim 2 \mathrm{Gyr}$, based on the MAI. Finally, Figure 7d shows the CMD for the cluster Berkeley 104 (Be 104). The presence of a likely red-giant clump at V 15.3, (V-I) 1.7 suggests that Be 104 too is an old cluster, with an age of $\sim 7-8 \mathrm{Gyr}$, based on the MAI. Berkeley 104 is, therefore, among the oldest open clusters known. 

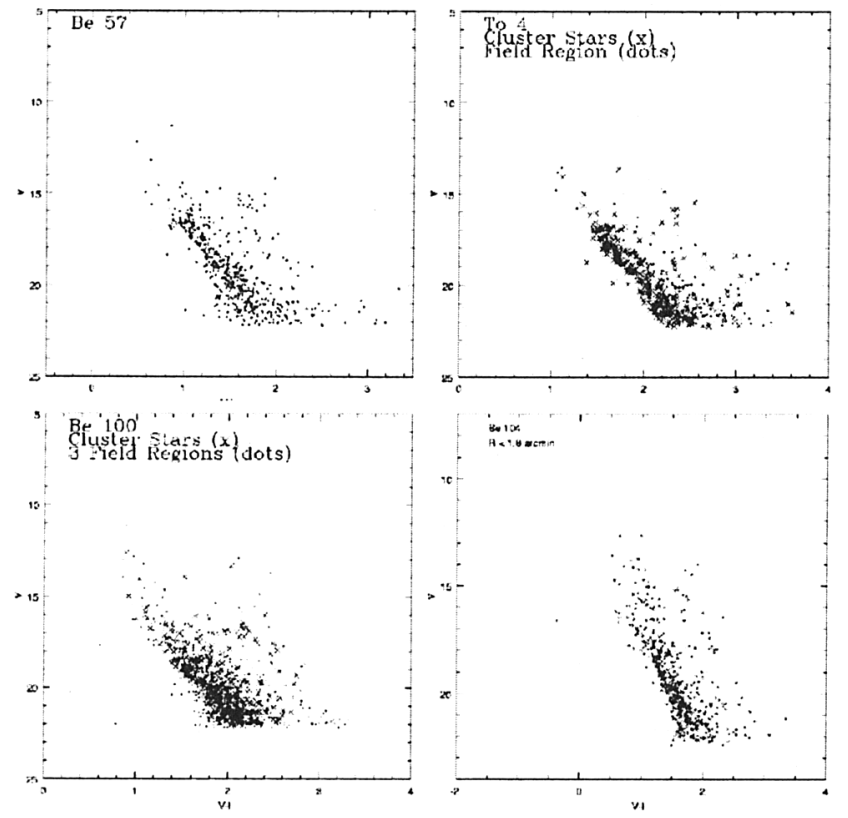

Figure 7. CMDs for four newly identified old open clusters. Figure 7a: Berkeley 57. Figure 7b: Tombaugh 4. Figure 7c: Berkeley 100. Figure 7d. Berkeley 104. See text for details.)

\section{Summary}

Although technology is allowing for the investigation of star clusters outside of the Milky Way, it is important to realize that a significant number of clusters in our own Galaxy remain poorly studied. If we are to fully understand the formation and evolution of star clusters, from small aggregates to super star clusters, and use clusters to probe the evolution of galaxies, it is essential that we devote effort to those clusters that lie within our own backyard. It is also important to realize that uncertainties in stellar evolution models, coupled with problems of field star contamination and the relative paucity of stars in many open clusters, may limit the precision to which information can be extracted from star clusters. Nevertheless, given the wealth of information available from them, Galactic open clusters still make rich targets for those who are willing to remain close to home, and study objects within their own backyard.

\section{References}

Anthony-Twarog, B. J. \& Twarog, B. A., 1985, ApJ, 291, 595

Bica, E., Ortolani, S. \& Barbuy, B. 1999, A\&AS, 136, 363

Buonanno, R., Corsi, C. E. \& Fusi Pecci, F. 1989, A\&A, 216, 80

Carraro, G., Chiosi, C., Bressan, A. \& Bertelli, G. 1994, A\&AS, 103,375 
Carraro. G. Girardi, L. \& Chiosi, C. 1999a, MNRAS, 309, 430

Carraro, G., Ng \& Portinari, 1998, MNRAS, 296, 1045

Carraro, G. \& Ortolani, S. 1994, A\&A, 291, 106

Carraro, G. \& Patat, F. 1995, MNRAS, 276, 563

Carraro, G., Vallenari, A., Girardi, L. \& Richichi, A. 1999b, A\&A, 343, 825

Friel, E. D. 1995, ARAA, 33, 381

Friel, E. D. \& Janes, K. A. 1993, A\&A, 267, 75

Geisler, D. 1987, AJ, 94, 84

Janes, K. A. \& Phelps, R. L. 1994, AJ, 108, 1773

Kalirai, J. S., Richer, H. B., Fahlman, G. G., Cuillandre, J.-C., Ventura, P., D'Antona, F., Bertin, E., Marconi, G. \& Durell, P. R. 2001, AJ, 122, 257

Kaluzny, J. 1994a, A\&AS, 108, 151

Kaluzny, J. 1994b, AcA, 44, 247

Kassis, M., Janes, K. A., Friel, E. D. \& Phelps, R. L. 1997, AJ, 113, 1723

Keller, A. C., Grebel, A. K., Miller, G. J. \& Yoss, K. M. 2001, AJ, 122, 248

Lapasset, E., Clari, J. J. \& Mermilliod, J.-C. 2000, A\&A, 361, 945

Lee, S. H., Kang, Y.-W. \& Ann, H. B. 1999, PKAS, 14, 61

Lyngå, G. 1987 Catalog of Open Cluster Data (5th edition), CDS, Strasbourg

Mathieu, R. D. 2000, Stellar Clusters and Associations: Convection, Rotation and Dynamos, eds. R. Pallavicini, G. Micela and S. Sciortino, ASP Conf. Ser., 198 , p. 517

Mermilliod, J.-C. 2000, Stellar Clusters and Associations: Convection, Rotation and Dynamos, eds. R. Pallavicini, G. Micela and S. Sciortino, ASP Conf. Ser., 198 , p. 513

Montgomery, K. A., Janes, K. A. \& Phelps, R. L. 1994, AJ, 108, 585

Montgomery, K. A., Marschall L. A. \& Janes, K. A. 1993, AJ, 106, 181

Patat, F. \& Carraro, G. 1995a, MNRAS, 272, 507

Patat, F. \& Carraro, G. 1995b, A\&AS, 114, 281

Perryman, M. A. C., Brown, A. G. A., Lebreton, Y., Gomez, A., Turon, C., de Strobel, G. C., Mermilliod, J.-C., Robichon, N., Kovalevsky, J. \& Crifo, F. 1998, A\&A, 331,81

Phelps, R. L. 1996, AJ, 111,1604

Phelps, R. L. 1997, ApJ, 483, 826

Phelps, R. L. 2000, in Chemical Evolution of the Milky Way: Stars Versus Clusters

Phelps, R. L. \& Janes, K. A. 1994, ApJS, 90, 31

Phelps, R. L., Janes, K. A. \& Montgomery, K. A. 1994, AJ, 107, 1079

Piatti, A. E., Claria, J. J. \& Bica, E. 2000, A\&A, 360, 529

Sandrelli, S., Bragaglia, A., Tosi, M. \& Marconi G. 1999, MNRAS, 309, 739

Sarajedini, A. \& Demarque, P. 1990, ApJ, 365, 219

Subramaniam, A. \& Sagar, R. 1999, AJ, 117, 937

Tosi, M., Pulone, L. Marconi, G. \& Bragaglia, A. 1998, MNRAS, 299, 834 
Twarog, B.A., Anthony-Twarog, B. J. \& Hawarden, T. G. 1995, PASP, 107, 1215

Twarog, B. A., Ashman, K. M. \& Anthony-Twarog, B. J. 1997, AJ, 114, 2556

van den Bergh, S. \& McClure, R. D. 1980, A\&A, 88, 360

VandenBerg, D. A., Bolte, M. \& Stetson, P. B. 1990, AJ, 100, 445

\section{Discussion}

R. Buta: I am curious about the detectability of old open clusters in other galaxies. They seem to be large enough to be resolvable in nearby galaxies, but what is known about their integrated absolute magnitudes?

R. Phelps: There are some estimates of integrated magnitudes in the Lynga catalog. The major constraint, I believe, is the sparse nature of the clusters, coupled with their lower brightnesses (particularly the older clusters I am discussing).

B. Harris: NGC 6791 is an extremely valuable cluster because it is both populous and old and metal-rich, so it provides a template for what the old red giant branch looks like at high (above solar) abundance. Is there any hope of finding any other such objects in these new surveys?

R. Phelps: I am skeptical that similar clusters will be found. There are, however, massive clusters that have been found in the Lynga catalog. As you know, IC 1257 was listed as an open cluster, but it is in fact a globular. It isn't the kind of cluster you asked about but it suggests it is possible. It is important, too, to remember that the cataloged clusters represent only a small fraction of those that exist throughout the Galaxy, even though we can't see them.

B. Santiago: I noticed that Be 17 has a parallel sequence in the background of its CMD. Do you know what it is and what effect it may have on age determination?

R. Phelps: It is a result of field star contamination, but I do not currently have field region data to do a statistical subtraction. The cluster main-sequence, however, separates nicely from that sequence and I do not think it affects the age determination to any great extent, but it would be useful to correct for field star contamination to confirm that statement.

G. Piotto: There is another interesting object at the borderline between open and globular clusters: Pal 1 . It is likely a globular cluster, but it has an age of $\sim 8$ Gyr, as shown in Rosenberg et al. (1999).

R. Phelps: It is an object that should be plotted on the histogram of cluster ages, but the figure I showed was an older one. Thak you for pointing that out.

G. Wallerstein: Your figure showing the Galactic distribution of clusters shows almost no clusters closer to the Galactic center than about half of the solar dis- 
tance. Is that real or due to a lack of recognition in that region?

R. Phelps: It is probably real.

H. Zinnecker: How uniform are the star-to-star metallicities within a given Galactic open cluster?

E. Friel: From abundance studies of red giants in the old open clusters and $\mathrm{F}$ and $\mathrm{G}$ dwarfs in some younger clusters, there does not appear to be any star-to-star variation in metallicities within a cluster. Abundances appear homogeneous, within observational errors. Cluster-to-cluster variations are, however, significant and show inhomogeneity in metallicity at a given age or position in the Galactic disk.

J. Palous: The young open clusters: do they move closer to circular orbits around the Galactic center than the old open clusters?

R. Phelps: The data that are available are consistent with the old open clusters having circular, or near circular orbits. I don't think one can be more specific than that.

F. D'Antona: I have to comment that the turnoff of Be 17 seems to be at the borderline between stars having a convective core or not, and it is quite difficult to model whether there is any, and the extent of, core overshooting. The resulting modelization is also dependent on the metallicity, which must be very well known before we draw conclusions.

R. Phelps: I certainly agree. I hope further study of Be 17 will provide that type of information. 